\title{
Katalog der in den Sammlungen des Senckenberg Deutschen Entomologischen Instituts aufbewahrten Typen - Coleoptera: Scolytidae (Curculionoidea)
}

\author{
LUtz Behne ${ }^{1}$ und HANnelore GaEdiKe ${ }^{2}$
}

\author{
${ }^{1}$ Senckenberg Deutsches Entomologisches Institut, Eberswalder Straße 90, 15374 Müncheberg \\ ${ }^{2}$ Florusstraße 5, 53225 Bonn \\ Published on 2013-12-20
}

\section{Zusammenfassung}

Die Typen der Familie Scolytidae (Coleoptera, Curculionoidea) in den Sammlungen des Senckenberg Deutschen Entomogischen Instituts werden aufgelistet. Mitgeteilt werden jeweils das Zitat der Erstbeschreibung und Angaben uber das vorhandene Typenmaterial. Die Sammlungen enthalten 155 Primartypen, davon 50 Holotypen und Syntypen von 105 Arten. Von 46 weiteren Arten existieren Paratypen oder Paralectotypen. Von 12 Arten sind als Typen etikettierte Tiere wahrscheinlich nicht beschrieben.

\section{Summary}

The type specimens of the family Scolytidae (Coleoptera, Curculionoidea) in the collections of the Senckenberg Deutsches Entomologisches Institut are listed. In each case, the original description is cited and information on the extant type material is presented. The collections contain 155 primary types, including 50 holotypes and syntypes of 105 species. Paratypes and paralectotypes of a further 46 species are present. Specimens of 12 species are labelled as types, but these species have probably not been described.

\section{Scolytidae}

\section{aceris LINDEMANN, 1875, Dryocoetes}

Bull. Mosc. 49 (1), 140-143

Mehrere Syntypen, bei Moskau, leg. Lindemann, Anf. X. SDEI: 3 Syntypen, Mosqua, leg. Lindemann-Kiesenwetter, Coll. v. Heyden und Coll. KraAtz.

\section{aceris KNOTEK, 1892, Scolytus}

Wien. ent. Ztg. 11, 235-236

Mehrere Syntypen, Bosna, Herzegovina, Austria inferior, durch KNOTEK.

SDEI: 1 Syntypus, Ruiste bei Mostar, leg. KnOTEK. aegir EgGens, 1922, Xyleborus

Ent. Bl. 18, 171

Mehrere Syntypen, Deutsch-Ostafrika, im SDEI und in Coll. EgGers.

SDEI: 1 Syntypus, Ostafrika, Coll. KRAATz.

\section{aethiopicus Eggens, 1936, Triotemnus}

Ann. Mag. Nat. Hist. (10) 18, 31

$0^{7}$ Holotypus, 18 Paratypen, Abyssinia, Jem-Jem Forest, circa 8000 ft., 26.IX.1926, leg. Sсотт, im Britischen Museum und in Coll. Eggers.

SDEI: 1 Paratypus, mit den Angaben der Beschreibung (Djem-Djem), ex Brit. Mus. 1927-127, from decayed parts of tree-Euphorbia. 


\section{affinis StrohmeYER, 1911, Cladoctomus}

Ent. Bl. 7, 17-18, Fig. 1, 2

Holotypus, Abessynien, Aethiopia, in Coll. Strohmeyer [Harrar].

\section{alni LindeMANn, 1875, Cryphalus}

Bull. Mosc. 49 (1), 136-140

Mehrere Syntypen, bei Moskau, auf Alnus incana, E.V. oder A.I.

SDEI: 9 Syntypen, Mosqua, leg. Lindemann: Coll. KraAtz, Coll. Letzner; Coll. Koltze; Coll. v. Heyden, durch v. Kiesenwetter.

\section{alni Pfeffer, 1940, Taphrorychus}

Acta Soc. Ent. Boh. (Časopis) 37, 53, Foto

Mehrere Syntypen; Gallia merid., Agay; Corsica, Ajaccio, V.1929.

SDEI: $2{ }^{\top}$ Syntypen, Agay Fr., V.1928, leg. Pfeffer (nicht 1929 !).

\section{amphicranoides HAGEDORN, 1908, Xyleborus}

Dtsch. ent. Ztschr. 1908, 379-380, Fig. 23

Mehrere Syntypen, Sumatra, Si-Rambé, Mentawei-SiOban, leg. Modigliani, im SDEI.

SDEI: 2 Syntypen, Sumatra, Si-Rambé, XII.1890-III.1891, leg. Modigliani, Coll. KraAtz.

\section{annectens Wo0d, 2007, Campocerus}

Bark and ambrosia beetles of South America (Coleoptera, Scolytidae). Brigham Young University, M.L. Bean Life Science Museum; Provo, Utah, 900 p., Beschr. p. 210.

Holotypus, o ${ }^{\star}$, Brazil, Serra de Baturite (Céara), L. Grounelle leg., I.1895, im SDEI, Coll. STrohmeYER.

armiger SCHEDL, 1953, Xyleborus

Ent. Bl. 49, 28

Mehrere Syntypen, China, Fukien, Kuatun, 21., 25.IV. 1946, leg. Tschung Sen, in Coll. Klapperich und Coll. SCHEDL.

SDEI: 1 Syntypus, mit den Angaben der Beschreibung, 21.IV.1946.

artecomans SCHEDL, 1953, Xyleborus

Ent. Bl. 49, 23-25, Abb. Nr. 1

Mehrere Syntypen, China, Fukien, Kuatun, 2300 m, 27,40 N., 117,40 E., 21.V.1938, Coll. Klapperich; Kuatun, 7., 26., 27.VIII.1946, leg. Tschung SEN; in Coll. KLAPPERICH und Coll. SCHEDL.

SDEI: 1 Syntypus, China, Fukien, Kuatun, 15.VIII.1946, leg. TSCHUNG SEN.

\section{aspericollis StROHMEYER, 1909, Phloeoborus}

Ent. Bl. 5, 248

o Holotypus, Ecuador, Llanos, Coll. Strohmeyer, im SDEI. ater EGGERS, 1933, Ozopemon

Ent. Nachr. Bl. 7, 101

Holotypus, mehrere Paratypen, China, Szechuan, Mukue Tatsienlu, in Coll. Eggers und Coll. ReITTER.

SDEI: 4 Syntypen, China, Szechuan, Mukue Tatsienlu, durch ReITTER.

\section{ater EgGERS, 1936, Thamnurgides}

Tjidschr. Ent. 79, 84

Holotypus, mehrere Ex. Sumatra, Brastagi, VII.1934, leg. PALM, an Mitrastemon spec. (Rafflesiaceae); im SDEI und in Coll. Eggers; 1 Ex. Malay Peninsula, Pahang: Paratypen.

SDEI: Holotypus, mit den Angaben der Beschreibung, 1924 (nicht 1934!); 1 Ex. dito; 1 Ex. Sumatra; Paratypen.

aterrimus Eggens, 1933, Hylastes

Ent. Bl. 29, 3-4

Mehrere Syntypen, Sibirien, Rußland, Bulgarien, in Coll. EGGERS.

SDEI: 3 Syntypen, Transbaikal, Werchne Udinsk, durch BIENER.

\section{aterrimus Strohmeyer, 1908, Polygraphus}

Ent. Wochenbl. Leipzig 25, Nr. 16, 69

4 Syntypen, Himalaya occidentalis, Kulu (Cedrus deodara), leg. Rost, in Coll. Strohmeyer, im SDEI [1906].

\section{auratum Hagedorn, 1910, Ctonoxylon}

Dtsch. ent. Ztschr. 1910, 4

Holotypus, Kamerun, leg. ConRadt, im SDEI [Coll. KRAATZ].

\section{balcanicus Eggers, 1911, Eccoptogaster}

Ent. Bl. 7, 75

Syntypen: mehrere Ex., bei Kievo, Umgebung von Sarajevo, 1902, leg. Apfelbeck; $1 \sigma^{\top}, 1$ ㅇ, dito, in Coll. EGGERs; $10^{\star}$, Bosnien, bei Bjelašnica-planina, leg. LEONHARD, in Coll. LEONHARD.

SDEI: 1 Syntypus, Bosnia, Bjelašnica-planina, leg. LEONHARD.

\section{barbatus HAGEDORN, 1909, Sphaerotrypes}

Dtsch. ent. Ztschr. 1909, 739, Fig. 33

1 Ex. Nordost-Sumatra, leg. Schultheiss; 1 Ex. Kamerun, leg. ConRADT; im SDEI: Syntypen.

SDEI: 1 Syntypus, N.O.-Sumatra, Tebing-tinggi, leg. Schultheiss, Coll. Schultheiss.

\section{barbatus HAGEDORN, 1910, Xyleborus}

Dtsch. ent. Ztschr. 1910, 11

3 Syntypen: Sumatra, leg. Foerster, in Coll. Hagedorn; Mentawei, leg. Modigliani, im SDEI.

SDEI: 2 Syntypen, Mentawei, Sipora, Sereinu, V.-VI.1894, leg. Modigliani, Coll. KraAtz. 
bicolor StROHMEYER, 1910, Kyrtogernius

Ent. Bl. 6, 127-128

Mehrere Syntypen, Aethiopia, Tschertscher Montes.

SDEI: 14 Syntypen, Aethiopia, Tschertscher Montes, 1910, leg. KRISTENSEN.

bicornis EgGers, 1923, Xyleborus

Zool. Meded. Mus. Leiden, 7, 194-195

2 Syntypen, Niederl. Indien, im SDEI und in Coll. EGGERS.

SDEI: 1 Syntypus, Holländ. Indien.

biseriatus EgGers, 1943, Corthylus

Arb. morph. tax. Ent. 10, 247

o Holotypus, Guatemala, leg. Conradt, Coll. StrohMEYER, im SDEI.

\section{biseriatus SCHEDL, 1963, Xyleborus}

Reichenbachia 1, 226-227

Holotypus, mehrere Paratypen, Brasilien, Santa Catarina, Nova Teutonia, IV.1956/XII.1955/VIII.-X.1957/ VIII.1958, leg. Plaumann, in Coll. Schedl, Coll. PlauMANN und im SDEI.

SDEI: 1 + Paratypus, Brasilien, Nova Teutonia, $27^{\circ} 11^{\circ}$ B., $52^{\circ} 23^{\circ}$ L., 300-500 m, X.1967, leg. Plaumann.

bituberculatum HAGEDORN, 1909, Rhopalopselion

Dtsch. ent. Ztschr. 1909, 740, Fig. 34

Holotypus, Kamerun, leg. ConRadT, im SDEI [Coll. KraATZ].

\section{bituberculatus HAGEDORN, 1910, Xylocleptes}

Dtsch. ent. Ztschr. 1910, 1

Holotypus, Kamerun, im SDEI (Type wahrscheinlich verloren).

\section{brasiliensis SCHEDL, 1940, Dryocoetes}

Arb. morph. tax. Ent. 7, 207

4 Syntypen, S. Paulo, leg. KRUG.

SDEI: 1 Syntypus, Brasil-S. Paulo, leg. KRUG.

brasiliensis SCHEDL, 1963, Xylocleptes

Reichenbachia 1, 224

Holotypus, 4 Paratypen, Brasilien, Santa Catarina, Nova Teutonia, 24.IX., 2., 9.X., 2.XI.1949/ 17.IV.1950, leg. Plaumann, in Coll. Schedl, Coll. Plaumann und im SDEI.

SDEI: 1 Paratypus, Brasilien, Nova Teutonia, $27^{\circ} 11^{\circ}$ B., $52^{\circ} 23^{\circ}$ L., 300-500 m, 2.XI.1909, leg. Plaumann, Morus nigra.

\section{brevicollis StroHmeYer, 1910, Acanthophorus}

Ent. Bl. 6, 70-71, Fig. 1-4

$10^{*}, 3$ \& Syntypen, Usambara ocidentalis, Mgila, leg. Karasek, in Coll. Strohmeyer.

SDEI: $1 \sigma^{\star}, 2$ ㅇ Syntypen, West-Usambara. buyssoni ReITTER, 1901, Pityophthorus

Wien. ent. Ztg. 20, 101

12 Syntypen, bei Brout-Vernet, leg. Du Buysson.

SDEI: 8 Syntypen, mit den Angaben der Beschreibung.

caesius HAGEDORN, 1909, Diamerus

Dtsch. ent. Ztschr. 1909, 735-736

Holotypus, Sumatra, leg. SchultheIss, im SDEI

[N.O. Sumatra, Tebing-tinggi, Coll. Schultheiss].

camerunum HAgEDoRn, 1910, Ctonoxylon

Dtsch. ent. Ztschr. 1910, 4, Fig. 42

Holotypus, Kamerun, leg. ConRadT, im SDEI [Coll. KRAATZ].

camerunus Hagedorn, 1909, Araptus

Dtsch. ent. Ztschr. 1909, 743

Holotypus, Kamerun, leg. ConRADT, im SDEI, Ex. wahrscheinlich verloren.

camerunus Hagedorn, 1908, Dactylipalpus

Dtsch. ent. Ztschr. 1908, 371, Fig. 5

Holotypus, Kamerun, leg. ConRadt, im SDEI [Coll. KRAATZ].

camerunus Hagedorn, 1908, Hypaspistes

Dtsch. ent. Ztschr. 1908, 374-375, Fig. 9-13

$10^{\star}, 1$ i Syntypen, Kamerun, leg. ConradT, im SDEI.

SDEI: 1 o Syntypus, Kamerun, leg. Conradt, Coll. KraATZ.

camerunus Hagedorn, 1909, Strombophorus

Dtsch. ent. Ztschr. 1909, 742

3 Syntypen, Kamerun, leg. Conradt, im SDEI.

SDEI: 1 Syntypus, Kamerun, leg. ConradT, Coll. KraAtz.

camerunus HagEDORN, 1910, Xyleborus

Dtsch. ent. Ztschr. 1910, 9

Mehrere Syntypen, Kamerun, leg. ConRAdT, im SDEI. SDEI: 3 Syntypen, Kamerun, leg. Conradt, Coll. KRAATZ.

canum ISRAELSON, 1990, Liparthrum

Bocagiana No. 137, 6-7

o Holotypus, Canary Is., La Palma, Lomo Caballo, $500 \mathrm{~m}$, Echium aculeatum, 13.6.1965, 352, leg. IsRAELson. Paratypen siehe Originalbeschreibung.

SDEI: 2 Paratypen, Ins. Canar., Gran Canaria, Costa de Silva, Montafia, 7.7.1971, leg. IsRAELSON.

caraibicus Eggens, 1941, Xyleborus

Arb. morph. tax. Ent. 8, 103

Holotypus, mehrere Paratypen, Guadeloupe, in Coll. EGgERs, im Museum Paris und SDEI.

SDEI: 1 Paratypus, Guadeloupe, leg. Dufau, 1909, Coll. Bonhoure, ex Museum Paris. 
carpini EgGeRs, 1942, Dryocoetes

Arb. morph. tax. Ent. 9, 32-33

Holotypus, 3 Paratypen, Ussuri, Lubarkyi, 20.IV.1931, durch STARCK, in Coll. EgGers und im SDEI.

SDEI: 1 Paratypus, Ussuri, Lubarkyi, 20.IV.1931.

cecropii SCHEDL, 1937, Hexacolus

Arb. morph. tax. Ent. 4, 66

Mehrere Syntypen, Costa Rica, La Hondura, $1450 \mathrm{~m}$, Coll. Nevermann, im SDEI, in Coll. SchedL und in Coll. NeVERMann.

SDEI: 1 đ̛, 2 ㅇ Syntypen, Costa Rica, San José, Hamburg Farm, 5.VI.1936, leg. NEVERMANN.

\section{cembrae Seltner, 1911, Polygraphus}

Zentralbl. Forstw. Wien 37, 99, 109, Fig. 10, 11, 12

Mehrere Syntypen, Steiermark, Tirol, 1910, leg. Seitner. SDEI: $20^{x}, 2$ o Syntypen, Styria, Hinterberg, 1.X.1910, leg. Seitner, Coll. Koltze.

\section{coffeae WURTH, 1908, Xyleborus}

Med. Proefst. Java te Salatiga, ser. 2, No. 3, 1-18, Taf. 1-3

Mehrere Syntypen, Mittel-Java, XI.1906.

SDEI: $2 o^{\star}, 3$ \& Syntypen, Java.

concolor HAgedorn, 1909, Hypothenemus

Dtsch. ent. Ztschr. 1909, 744, Fig. 40

3 Syntypen, Kamerun, leg. ConRadT, im SDEI.

SDEI: 1 Syntypus, Kamerun, leg. Conradt, Coll. KRAATZ.

\section{confusus Eggers, 1941, Mimips}

Arb. morph. tax. Ent. 8, 179

Holotypus, Spanien-Guinea, Fernando Poo, 14.IV.1940, leg. EIDMANN, im SDEI [ 9 , Bata, Lichtfang].

\section{conradti HagedoRn, 1910, Xyleborus}

Dtsch. ent. Ztschr. 1910, 8

2 Syntypen, Kamerun, leg. ConRADT, im SDEI.

cordatus Hagedorn, 1909, Strombophorus

Dtsch. ent. Ztschr. 1909, 741-742, Fig. 36

Holotypus, Kamerun, leg. ConRadt, im SDEI [Coll. KrAATZ].

cordatus Hagedorn, 1910, Xyleborus

Dtsch. ent. Ztschr. 1910, 12

Mehrere Syntypen, Mentawei, leg. Modigliani.

SDEI: 4 Syntypen, Mentawei, Sipora, Sereinu, V.-VI.1894, leg. Modigliani, Coll. KraAtz.

cornutum Eggers, 1943, Ctonoxylon

Arb. morph. tax. Ent. 10, 246-247

$\mathrm{O}^{\star}$ Holotypus, Kamerun, Coll. Strohmeyer, im SDEI. corsicus EgGERS, 1912, Trypophloeus

Ent. Bl. 8, 114-115

Holotypus, Corsika, Mont Renoso, leg. LeOnhard, in Coll. LeONHARD [1905].

corthyloides Hagedorn, 1910, Premnobius

Dtsch. ent. Ztschr. 1910: 1; als var. von cavipennis ЕІсHн. beschrieben

Holotypus, Kamerun, im SDEI.

\section{crenatum Hagedorn, 1910, Ctonoxylon}

Dtsch. ent. Ztschr. 1910, 5

Holotypus, Kamerun, leg. Conradt, im SDEI [Coll. KraATz].

\section{crenatus HagedoRn, 1909, Strombophorus}

Dtsch. ent. Ztschr. 1909, 740-741, Fig. 35

2 Syntypen, Kamerun, leg. ConRadT, im SDEI.

SDEI: 1 Syntypus, wahrscheinlich verloren.

crenatus Eggers, 1920, Xyleborus

Ent. Bl. 16, 42-43

5 Syntypen: Kangu, VII.1911, in Coll. Hagedorn; Kamerun, im SDEI und in Coll. EgGERs.

SDEI: 2 Syntypen, Kamerun.

crucifer HAgEDORN, 1908, Xyleborus

Dtsch. ent. Ztschr. 1908, 381

Holotypus, Kamerun, leg. ConRAdT, im SDEI [Coll. KRAATZ].

\section{cucurbitae SCHEDL, 1939, Dendrocanulus}

Arb. morph. tax. Ent. 6, 45-46

Mehrere Syntypen, Costa Rica, Hamburgfarm, ex Cucurbita mexicana, leg. NevermanN, im SDEI und in Coll. SCHEDL.

SDEI: 4 Syntypen, Costa Rica, San José, Hamburgfarm, leg. NevermanN.

\section{demaisoni Eggers, 1912, Eccoptogaster}

Ent. Bl. 8, 47-48

Holotypus, 6 Paratypen, Sicilia, Algeria, Hispania, in Coll. Demaison, Coll. Leonhard und Coll. Eggers.

SDEI: 2 Paratypen, Sicilia, Ficuzza, 1906, leg. G. KRÜGER, Coll. LEONHARD.

\section{denticulatus Wichmann, 1915, Trigonogenius}

Ent. Bl. 11, 106-107

Holotypus, Deutsch-Ostafrika, Coll. KraATz, im SDEI.

\section{dentifrons Blackman, 1921, Phthorophloeus}

Techn. Bull. Miss. Agric. Expt. Sta. 10, 3-4, Taf. 1, Fig. 1

Holotypus, mehr als 100 Paratypen, Mississippi, A. \& M. College, 23., 24.III., 19., 24.V.1920, leg. Blackmann und University of Kansas, Lawrence, Kans., 10.VI.1920.

SDEI: 1 Paratypus, Mississippi, Merscia H., 23.III.1920, leg. Blackman, N. Y. S. C. F., Lot M. 175. 
derelictus Hagedorn, 1910, Xyleborus

Dtsch. ent. Ztschr. 1910, 12

Holotypus, Ostafrika, im SDEI [Coll. KraAtz].

dimorphus SCHEDL, 1959, Pityophthorus

Beitr. Ent. 9, 551-552

Holotypus, mehrere Paratypen, Brasilien, Matto Grosso, Rio Caraguata, IV., V.1953, leg. Plaumann, in Coll. Schedl, Coll. Plaumann und im SDEI.

SDEI: 4 Paratypen, mit den Angaben der Beschreibung, IV.1953.

dubiosus SCHEDL, 1940, Hypothenemus

Arb. morph. tax. Ent. 7, 207

Mehrere Syntypen, Costa Rica, Hamburgfarm, Ebene Limon, 15.XI.1937, Coll. NevermanN, im SDEI und in Coll. ScHEdL.

SDEI: 4 Syntypen, mit den Angaben der Beschreibung.

dubius SCHEDL, 1959, Hylocurus

Beitr. Ent. 9, 547-548

Mehrere Syntypen, Brasilien, Rondon, IX., X., XI.1952, leg. Plaumann, in Coll. Schedl, Coll. Plaumann und im SDEI.

SDEI: $10^{*}, 1$ ㅇ Syntypen, Brasilien, Rondon, $24^{\circ} 38^{\circ} \mathrm{B}$, $54^{\circ} 07^{\circ}$ L, VIII., IX.1952, 500, leg. Plaumann.

dubius Eggens, 1923, Xyleborus

Zool. Meded. Mus. Leiden 7, 199-200

2 Syntypen, Niederl. Indien, Tjitjalangka (Java) in „Dadap“, im SDEI und in Coll. EgGERS.

SDEI: 1 Syntypus, Holländ. Indien.

ellipticus EgGers, 1934, Hexacolus

Ent. Bl. 30, 80

Holotypus, Costa Rica, Turrialba, im SDEI [durch Heyne].

eos SPESSIVTSEV, 1919, Hylesinus

Ent. Monthly Mag. 55, 248-249

Mehrere Syntypen, Vicinity of Vladivostok, 1912/1915, leg. Czerski, Delle, Berger; Manchuria, 1916, leg. VERBICKY.

SDEI: 1 Syntypus, Wladiwostok, ded. SpessiwzefF.

fallax EGGERS, 1915, Ips

Ent. Bl. 11, 96-97

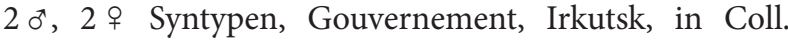
EGGERS.

SDEI: $1 o^{\star}, 1$ \% Syntypen, Gouv. Irkutsk, ded. SPESSIWZEFF.

fasciatus Hagedorn, 1909, Kissophagus

Dtsch. ent. Ztschr. 1909, 737

Mehrere Syntypen, Ostafrika, im SDEI.

SDEI: 5 Syntypen, Ostafrika, Coll. KraATz. fimbriatus SCHEDL, 1955, Gnathotrichus

Rev. Chil. Ent. 4, 259

Mehrere Syntypen, Chile, P. Arenas, Süd-Chile; Termas de Puyehue, leg. Schwabe; im SDEI, in Coll. Kuschel und Coll. SCHEDL.

SDEI: 2 ㅇ Syntypen, Süd-Chile, Puerto Puyuhuapi, 15.II., 7.XII.1939, leg. Schwabe, Nr. 499.

\section{flavescens HagedoRn, 1910, Ctonoxylon}

Dtsch. ent. Ztschr. 1910: 4; als var. von camerunum HAGEDORN beschrieben

Zahlreiche Syntypen, Kamerun, im SDEI.

SDEI: 2 Syntypen, Kamerun, leg. ConRADT.

\section{formosanus Eggers, 1939, Scolytus}

Arb. morph. tax. Ent. 6, 115-116

Holotypus, mehrere Paratypen, Formosa, X.1934, leg. SAIto, in Coll. Schedl und im SDEI.

SDEI: $1 \sigma^{\star}$ Paratypus, Formosa, X.1924, leg. SAIto.

\section{fuscicollis HAGEDORN, 1910, Ozopemon}

Dtsch. ent. Ztschr. 1910, 3

Syntypen: 2 Ex. Java; 1 Ex. Sumatra, leg. Foerster, in Coll. Hagedorn; 1 Ex. Sumatra, leg. Modigliani, im SDEI.

SDEI: 1 Syntypus, Mentawei, Sipora, Sereinu, V.-VI.1894, leg. Modigliani, Coll. KraAtz.

\section{fusciseriatus EgGens, 1934, Xyleborus}

Ent. Bl. 30, 82-83

+ Holotypus, Costa Rica, La Caja, 8 km westlich San José, leg. H. SCHMIDT, im SDEI.

fuscum Hagedorn, 1910, Ctonoxylon

Dtsch. ent. Ztschr. 1910, 5

Holotypus, Kamerun, leg. Conradt, im SDEI [ohne Fundort, Coll. KraATz].

gaunersdorferi ReITTER, 1885, Crypturgus

Dtsch. ent. Ztschr. 29, 389-390

Mehrere Syntypen, Euboea, leg. Emge.

SDEI: 2 Syntypen, Euboea, durch ReITTER.

globusus Strohmeyer, 1910, Glochiphorus

Ent. Bl. 6, 127, Fig. 3a-d

Mehrere Syntypen, Madagaskar, in Coll. Strohmeyer. SDEI: 7 Syntypen, Plateau de l'Androy-Reg. d'Ambovombe.

\section{gracilior SCHEDL, 1959, Pterocyclon}

Beitr. Ent. 9, 553-554

Holotypus, mehrere Paratypen, Brasilien, Nova Teutonia, IV.1944, leg. Plaumann, in Coll. Schedl, Coll. PlauMANN und im SDEI.

SDEI: 5 Paratypen, mit den Angaben der Beschreibung. 
granulatus Eggers, 1943, Phloeoborus

Arb. morph. tax. Ent. 10, 243-244

Holotypus, Franz. Guyana, Maroni, Coll. Strohmeyer, im SDEI.

\section{guineensis EGGERS, 1941, Xyleborus}

Arb. morph. tax. Ent. 8, 179-180

Holotypus, mehrere Paratypen, Insel Fernando Poo, Spanisch-Guinea, 3.II.1940, leg. SCHLÜTER, im SDEI und in Coll. EgGERs.

SDEI: ${ }^{\top}$ Holotypus, 1 ㅇ Paratypus, mit den Angaben der Beschreibung, Nr. 251.

\section{hagedorni Eggers, 1920, Sphaerotrypes}

Ent. Bl. 16, 122

Holotypus, Kamerun, leg. ConRADT, im SDEI.

hauseri ReItTeR, 1895, Ips

Verh. Nat. Ver. Brünn 33 (1894), 81

Mehrere Syntypen, südl. Turkestan, Thian-Schan, via Hauser an Reitter.

SDEI: 9 Syntypen, Thian-Schan, Musart, VI.1894, durch Hauser, Coll. Hauser und Coll. v. Heyden.

\section{henscheli SEITNER, 1887, Pityophthorus}

Wien. ent. Ztg. 6, 44-45

Mehrere Syntypen, Tirol, Frühjahr und Sommer, in den nächst Innsbruck und Hall gelegenen Zirbenbeständen.

SDEI: 4 Syntypen, Tirol, leg. SeItner, Forstadjunct i. Hall, Coll. STIERLin.

\section{herbellae Strohmeyer, 1929, Pityogenes}

Ent. Bl. 25, 181-182, Abb. 1, 2

Mehrere Syntypen, Spanien, Sierra de Guadarrama, ca. $2200 \mathrm{~m}$, leg. StrohmeYer.

SDEI: $20^{\star}, 1$ ㅇ Syntypen, Sierra de Guadarrama, 2400 m, VII.1927, Pinus silvestris, Coll. Strohmeyer.

\section{hercegovinensis EgGers, 1922, Phloeosinus}

Ent. Bl. 18, 120-121

8 Syntypen, Herzegowina, durch Tredl an Eggers.

SDEI: $1 \sigma^{\star}, 1$ \& Syntypen, Herzegowina.

\section{heydeni EICHHOFF, 1884, Tomicus}

Dtsch. ent. Ztschr. 28, 298-299

Mehrere Syntypen, Amur, Chabarofka.

SDEI: 6 Syntypen, Amur, Chabarofka, in Coll. v. Heyden und Coll. Koltze.

\section{himalayensis StROHMEYER, 1908, Dryocoetes}

Ent. Wochenbl. Leipzig 25, Nr. 39, 161

Mehrere Syntypen, westl. Himalaja, Kaschmir, leg. Rost. SDEI: 1 Syntypus, Kaschmir, Pir Panjal, leg. Rost, Coll. STROHMEYER.

\section{hirsutum Hagedorn, 1910, Ctonoxylon}

Dtsch. ent. Ztschr. 1910: 4; als var. von camerunum HAGEDORN beschrieben

Zahlreiche Syntypen, Kamerun, leg. ConradT, im SDEI. SDEI: 6 Syntypen, Kamerun, leg. ConraDT.

holtzi StrohmeYer, 1907, Thamnurgus

Wien. ent. Ztg. 26, 6

Holotypus, Graecia, nahe Kalàvryta, Morea, leg. Holtz [Coll. Strohmeyer].

\section{hylurgulus Eggens, 1940, Phloeosinus}

Ent. Bl. 36: 62 nom. nov. pro Hylurgulus sumatranus EGGERs, 1927; Treubia 9: 392

Siehe diese.

impar var. nanus HAGEDoRN, 1909, Diamerus

Dtsch. ent. Ztschr. 1909: 734

Holotypus, Togo, leg. ConRADT, im SDEI.

SDEI: Holotypus verloren gegangen.

infans HAgEDORN, 1910, Xyleborus

Dtsch. ent. Ztschr. 1910, 7

Mehrere Syntypen, Mentawei, leg. ModigLIAni, im SDEI. SDEI: 3 Syntypen, Mentawei, Sipora, Sereinu, V.-VI.1894, leg. Modigliani.

insularis Eggers, 1940, Cnesinus

Arb. morph. tax. Ent. 7, 138

Holotypus, mehrere Paratypen, Guadeloupe, Courbeyre, Environ de Trois-Riviéres, leg. Dufau, in Coll. Eggers, im Museum Paris und SDEI.

SDEI: 1 Paratypus, LA Guadeloupe, V., leg. Dufau, Coll. Bonhoure, 1909, Museum Paris.

\section{insularis EgGers, 1940, Coccotrypes}

Arb. morph. tax. Ent. 7, 129

Holotypus, mehrere Paratypen, Guadeloupe, Trois-Riviéres und Gourbeyre, Martinique, in Coll. Fleutiaux, Coll. EGGERs und im SDEI, an „branchettes de cacaoyer“. SDEI: 1 Paratypus, Martinique, 1901, leg. Fleutiaux.

\section{insularis Eggers, 1940, Dryocoetes}

Arb. morph. tax. Ent. 7, 127

Holotypus, mehrere Paratypen, Guadeloupe, Trois-Riviéres, Coll. Fleutiaux; Martinique, St. Pierre, 1902, leg. WADDY, im Museum Paris, im SDEI und in Coll. EgGERs. SDEI: 1 Paratypus, Martinique, St.-Pierre, 1902, leg. WADDY, ex Museum Paris.

\section{insularis Eggers, 1940, Neopityophthorus}

Arb. morph. tax. Ent. 7, 130-131

Holotypus, mehrere Paratypen, Guadeloupe, Trois-Riviéres, in Coll. Eggers, im Museum Paris, in Coll. HustaCHE und im SDEI.

SDEI: 2 Paratypen, Guadeloupe, Coll. Vitras. 
insularis Eggers, 1940, Phloeotribus

Arb. morph. tax. Ent. 7, 123-124

` Holotypus, mehrere Paratypen, Guadeloupe, 3 Riviéres, in Coll. Eggers, Coll. Fleutiaux, im Museum Paris und SDEI.

SDEI: 1 Paratypus, La Guadeloupe, V., leg. Dufau, Coll Bonhoure, 1909, ex Museum Paris.

\section{insularis EGGERS, 1932, Problechilus}

Wien. ent. Ztg. 49, 232-233

Holotypus, mehrere Paratypen, Guadeloupe, Trois Riviéres, im Museum Paris und in Coll. EgGers.

SDEI: 1 ㅇ, Paratypus, mit den Angaben der Beschreibung, leg. Dufau, Coll. Fleutiaux.

\section{insulindicus EgGers, 1923, Xyleborus}

Zool. Meded. Leiden 7, 177-178

2 Ex. Neuguinea (Südost), Paumomufluß, 1 Ex. S. O. N. G. Moroka; 2 Ex. Australien, Kuranda; im Museo Civico Genova und in Coll. EgGers; Syntypen.

SDEI: 2 Syntypen, Kurunda, Cairns, leg. Hacker, Coll. HACKER.

interruptus SCHEDL, 1959, Hylocurus

Beitr. Ent. 9, 548-549

Holotypus, mehrere Paratypen, Brasilien, Nova Teutonia, XII.1940, leg. Plaumann, in Coll. Schedl, Coll. PlauMANN und im SDEI.

SDEI: $2 \sigma^{\star}$ Paratypen, Brasilien, Nova Teutonia, XII.1940, Coll. Plaumann.

\section{kasukumbi SCHEDL, 1957, Polygraphus}

Ann. Mus. Congo Belge (Sér. 8E), Sci. zool. 56, 40-41

Holotypus, mehrere Paratypen, Congo Belge, Luki, 25.V.1952, 5., 7., 10.VI.1952, leg. SchedL, im Musée Royal du Congo Belge und in Coll. SchedL.

SDEI: 1 đ, 1 ㅇ Paratypen, Congo Belge, Luki, 25.V.1952, leg. SCHEDL, Nr. 87.

klapperichi SCHEDL, 1953, Poecilips

Ent. Bl. 49, 26-27

Mehrere Syntypen, China, Fukien, Kuatun, 29.XI.1946, leg. Tschung Sen, in Coll. Klapperich und in Coll. SCHEDL.

SDEI: 1 Syntypus, mit den Angaben der Beschreibung, 25.IV.1946, leg. TsCHUNG SEN.

\section{knoteki ReItTer, 1898, Pityophthorus}

Dtsch. Ent. Ztschr. 1898, 356-357

Mehrere Syntypen, Tirol, am Krummholz, via SEITner an ReITTER.

SDEI: 1 o Syntypus, Südtirol, Paneveggio, Pinus cembra, Coll. M. Seitner.

\section{koltzei ReITTER, 1894, Scolytus}

Wien. ent. Ztg. 13, 128

Mehrere Syntypen, Chingan, Amur, via Koltze an ReitTER.
SDEI: 7 Syntypen, Chingan, Amur, 1884, durch KoltzeReitter, Coll. Koltze und Coll. v. Heyden.

\section{kraatzi EıсHнHOF, 1864, Hylesinus}

Berl. ent. Ztschr. 8, 30

5 Syntypen, Pesth, leg. KraAtz.

SDEI: 2 Syntypen, Pesth, leg. KraAtz, Coll. KraAtz.

\section{kuennemanni REITTER, 1902, Hypothenemus}

Wien. ent. Ztg. 21, 140-141

Mehrere Syntypen, Bremen, in Paranüssen, leg. Altken, via Künnemann an ReitTer.

SDEI: 5 Syntypen, mit den Angaben der Beschreibung, 10.XII.1900, Coll. Künnemann, Coll. Leonhard.

\section{kunula StroHmeYer, 1908, Scolytoplatypus}

Ent. Wochenbl. Leipzig 25, Nr. 39, 161

o Holotypus, Kashmir, leg. Rost, in Coll. Strohmeyer.

laeve EGgERS, 1939, Trypodendron

Arb. morph. tax. Ent. 6, 122-123

$2 o^{\star}, 3$ i Syntypen, Japan, Karafuto, leg. Kinoshita, in Coll. EgGERs und im SDEI.

SDEI: 1 \& Syntypus, Japan, Karafuto.

\section{laevis StrohmeYer, 1911, Ozopemon}

Philipp. Journ. 6, 22-23, Fig. 4-6

+ Holotypus, 1 i Paratypus, Mindoro, Calapan, P. I., leg. WebB, Nr. 1421, in der Entomologischen Sammlung des Bureau of Science, Manial, P. I. und in Coll. Strohmeyer. SDEI: 1 + Paratypus, Mindoro, Calapan, leg. WebB, Acc. No. 1421, Lot Govt. Lab. Coll.

\section{langstoni BLACKMAN, 1920, Micracis}

Techn. Bull. Miss. Agric. Expl. Sta. 9, 26, Taf. 1, Fig. 5, Taf. 2, Fig. 17, Taf. 5, Fig. 45, 46

+ Holotypus, 89 Paratypen, Agricultural College, Mississippi, 9.II.23., 24.III., 12.V.1920, leg. BLACKMAN, in Coll. BlaCKMan, im Mississippi Agricultural College und im New York State College of Forestry.

SDEI: 1 Paratypus, A.M. Miss., 23.III.1920, N. Y. S. O. F., Lot Mi 185, leg. Blackman.

\section{lenkoranus ReITTER, 1913, Taphrorychus}

Wien. ent. Ztg. 32 (Beiheft), 96

Mehrere Syntypen, Kaspisches Meergebiet, in den Gebirgen von Talysch, bei Lyrik und Lenkoran, leg. LEDER.

SDEI: 3 Syntypen; Caspi.-M.-Gebiet, Lyrik, leg. LedER, Reitter; Lenkoran, leg. Leder, Reitter; Coll. StrohMEYER.

\section{leonhardi Eggens, 1912, Dryocoetes}

Ent. Bl. 8, 49

Mehrere Syntypen, Bulgaria, obere Iskertal, Rhilatal, V., VI.1911, leg. Hilf, in Coll. LeONHARD und Coll. StroHMEYER.

SDEI: 2 Syntypen, Bulgarien, obere Iskertal, V., 6.VI.1911, leg. Hilf, Coll. LeOnHARD. 
leonii EGGERS, 1908, Eccoptogaster

Naturalista Sicil., 2 (Separatabdruck)

Mehrere Syntypen, Italia, prov. Aquilensi, nahe Cerchio, leg. LEONI, in Coll. EgGERs.

SDEI: $10^{\star}, 1$ ㅇ Syntypen, Italia, Cerchio, leg. LEONI.

limbatus Eggers, 1943, Sphaerotrypes

Arb. morph. tax. Ent. 10, 244

Holotypus, mehrere Paratypen, Indien, Coll. STroHMEYER, im SDEI und in Coll. EgGERs.

SDEI: Holotypus, 10 Paratypen, Indien, Coll. STroHMEYER.

\section{longipilis SCHEDL, 1957, Polygraphus}

Ann. Mus. Congo Belg. (Sér. 8E), Sci. zool. 56, 35-36

Holotypus, mehrere Paratypen: Congo Belge, HembeBitale, 8., 12., 16., 19.VIII.1952; Yangambi, 4.VII., 13., 16.IX.1952, leg. SchedL; im Musée Royal du Congo Belge und in Coll. SCHEDL.

SDEI: $1 \sigma^{\star}, 1$ ㅇ Paratypen, Congo Belge, Hembe Bitale, 8.VIII.1952, leg. SCHEDL, Nr. 593.

\section{lunulatus Eggers, 1943, Phloeoborus}

Arb. morph. tax. Ent. 10, 242-243

$0^{\star}$ Holotypus, 1 , Brasil, Jatahy, im Staate Goyaz, in Coll. Strohmeyer, im SDEI; mehrere $\sigma^{*}$, dito und Mexico, Coll. Strohmeyer und im SDEI; $1 \sigma^{\star}$, Panama, Bugaba, durch Blandford; $10^{\star}, 1$ ㅇ, Peru, Marcapata, in Coll. EgGers: Paratypen.

SDEI: ${ }^{*}$ Holotypus, Goyaz, Jatahy, Coll. Strohmeyer; 6 Ex. dito; 4 Ex. Mexico; 1 Ex. ohne Fundort, Coll. STrohmeYer; Paratypen.

\section{luteus Hagedorn, 1919, Diamerus}

Dtsch. ent. Ztschr. 1919, 735

Holotypus, Sumatra, leg. Schultheiss, im SDEI [N. O. Sumatra, Tebing-tinggi, Coll. Schultheiss].

\section{maiche Eggers, 1942, Xyleborus}

Arb. morph. tax. Ent. 9, 36

Holotypus, mehrere Paratypen, Ussuri, VII.1931, leg. Kurenzow, durch Starck, in Coll. Eggers und im SDEI. SDEI: 1 Paratypus, Ussuri, VI.1931, leg. Kurenzow, Ulmus.

\section{major EgGERS, 1943, Blastophagus}

Ent. Bl. 39, 50-51

Holotypus, 1 Ex. Japan, leg. Kobe, in Coll. Eggers; 2 Ex. Nord-Korea, Seishin-Olto, im SDEI; 1 ㅇ, Kiautschou, im Museum Hamburg: Paratypen.

SDEI: 1 Ex. Black Pipe, 17.V.1928, leg. KoвE; 1 Ex. NordKorea, Seishin-Olto, leg. KricheldorfF: Paratypen.

\section{major StrohmeYer, 1911, Cyrtogenius}

Ent. Bl. 7, 16-17

Holotypus, Aethiopia, Abessynien, in Coll. Strohmeyer, im SDEI [Harrar, leg. KRISTENSEN]. major StrohmeYeR, 1911, Ozopemon

Philipp. Journ. 6, 23-24

Holotypus, No. 12007, 1 Paratypus, Luzon, Baatan, Limay, P. I., leg. Alvarez, in der Entomologischen Sammlung des Bureau of Science, Manila, P.I. und in Coll. StroHMEYER.

SDEI: 1 Paratypus, Philippinen, Bataan, Limay, Acc. Nor. 12007, Lot Bu. of Sci., P. I., leg. Alvarez.

\section{mandschuricus SCHEDL, 1941, Scolytus}

Ent. Bl. 37, 42-43

Mehrere Syntypen, Mandschurei, Weischache, V.1938/ 10.-15.VII.1939, Coll. Alin, in Coll. Frey, Coll. SchedL und im SDEI.

SDEI: 2 Syntypen, Mandschuria centr., Weischache, 10.15.VI.1939 (nicht 15.VII.!), ded. AlIN.

\section{marginatus HAGEDORN, 1912, Xestips}

Dtsch. ent. Ztschr. 1912, 353-354

Holotypus, Ostafrika, im SDEI.

SDEI: Holotypus, wahrscheinlich verloren.

\section{marmoratus StrohmeYeR, 1914, Dactylipalpus}

Ent. Bl. 10, 73-74

$10^{\star}$, Deutsch-Ostafrika, Kolassini; $10^{\star}, 1$ , Natal: Syntypen.

SDEI: 1 Ex. Kolassini; 1 Ex. Natal, durch SAunders; Coll. STrohmeyer, Syntypen.

\section{meridianus Blackman, 1920, Micracis}

Techn. Bull. Miss. Agric. Expl. Sta. 9, 29-30, Taf. 1, Fig. 7, 8, Taf. 2, Fig. 19, Taf. 5, Fig. 43

Holotypus, 67 Paratypen, Corinth, Ripley, Wallerville und Agricultural College Miss., 1920, in Coll. BLACKMan, Coll. Mississippi Agricultural College und New York State College of Forestry at Syracuse.

SDEI: 1 Paratypus, A. M., 24.IX.1920, N.Y.S.C.F., Lot Mi 414 , leg. BLACKMAN.

\section{meridionale EgGeRs, 1940, Trypodendron}

Zentr.bl. f. Forstw. Wien 66, 38-39

1 , Rumänien, Biharfüred, leg. Boкor; 2 , Rodnaer Gebirge, leg. Deubel; 1 Ex. Frankreich, Alpes Maritimes, Thorenc, leg. Normand; 2 ㅇ, Jugoslawien, Sarajevo, Pl. Johorina, leg. Apfelbeck; in Coll. EgGers; $q$ Holotypus, mehrere Ex. Kleinasien, Ayancik, leg. ScHImitscheK, in Coll. Eggers und Coll. SchimitscheK: Paratypen.

SDEI: $1 \sigma^{*}$, Paratypus, Asia minor, Ayancik, leg. SchimitSCHEK.

\section{mexicanus BlANDF0RD, 1897, Dendroterus}

Biol. Centr. Amer. 4 (6), Col. 233, Taf. 7, Fig. 25

Mehrere Syntypen, Mexico, Tahuan tepec in Oaxaca, leg. HöGE.

SDEI: 1 Syntypus, Mexico, leg. HöGE. 
minimus Hagedorn, 1919, Chlortastus

Dtsch. ent. Ztschr. 1919, 738

3 Syntypen, Kamerun, leg. ConradT, im SDEI [Coll. KraAtz].

nanus Eggers, 1920, Coccotrypes

Ent. Bl. 16, 33

Mehrere Syntypen, Kamerun, leg. Conradt, in Coll. HAgEdorn, Coll. EgGers und im SDEI.

SDEI: 9 Syntypen, Kamerun, leg. ConRadT, Coll. KraATZ.

nitidus Eggers, 1933, Ips

Ent. Nachr.bl. Troppau 7, 101-102

Holotypus, mehrere Paratypen, China, Szechuan, Nitou Tatsienlu, Mukue Tatsienlu, in Coll. Eggers und Coll. REITTER.

SDEI: 7 Paratypen, China Szechuan, Nitou Tatsienlu, leg. REITTER.

\section{notatus Eggers, 1940, Hexacolus}

Arb. morph. tax. Ent. 7, 133

Holotypus, mehrere Paratypen, Guadeloupe, Trois-Riviéres, leg. Dufau, in Coll. Eggers, im Museum Paris und SDEI.

SDEI: 1 Paratypus, mit den Angaben der Beschreibung, 1904, ex Museum Paris.

notatus EgGers, 1941, Xyleborus

Arb. morph. tax Ent. 8, 107

Holotypus, mehrere Paratypen, Mittelamerika, Guadeloupe, Cuba, Habaña, San Dominica, Moca, Panama, Zativa, Volcan Chiriqui, Costa Rica, in Coll. Eggers und im SDEI.

SDEI: 1 Paratypus, Guadeloupe, Coll. VitraE.

obanus Hagedorn, 1910, Ozopemon

Dtsch. ent. Ztschr. 1910, 3

Mehrere Syntypen, Mentawei Si-Oban, leg. Modigliani, im SDEI.

SDEI: 1 Syntypus, Mentawei Si-Oban, IV.-VIII.1894, leg. Modigliani, Coll. KraAtz.

oblongus EgGers, 1940, Hexacolus

Arb. morph. tax. Ent. 7, 134-135

Holotypus, mehrere Paratypen, Guadeloupe, Env. de Trois-Riviéres, leg. Dufau, 1904, im Museum Paris, SDEI und in Coll. EgGERs.

SDEI: 1 Paratypus, mit den Angaben der Beschreibung, ex Museum Paris.

\section{obscuriceps Wood, 2007, Scolytus}

Bark and ambrosia beetles of South America (Coleoptera, Scolytidae). Brigham Young University, M.L. Bean Life Science Museum; Provo, Utah, 900 p., Beschr. p. 235.

Holotypus, 3 Paratypen, Brasilien, Rio Caragutá, $21^{\circ} 48^{\prime}$ Breite $52^{\circ} 27^{\prime}$ Länge, 400 m, 1953, leg. F. PlaumanN, im SDEI. obtusum EgGeRs, 1939, Trypodendron

Arb. morph. tax. Ent. 6, 121-122

Holotypus, mehrere Paratypen, Japan, Karafuto, Nopporo, in Coll. EgGERs und im SDEI.

SDEI: 1 o $^{\star}$ Paratypus, Simril, 25.VI.1928 (Fundort nicht lesbar!).

opacicollis Eggens, 1940, Chramesus

Arb. morph. tax. Ent. 7, 124-125

Holotypus, mehrere Paratypen, Guadeloupe, Environ de Trois-Riviéres, 1904, leg. Dufau, im Museum Paris, SDEI und in Coll. EgGERs.

SDEI: 1 \& Paratypus, Guadeloupe, Trois-Riviéres, Coll. VITRAE.

opacicollis var. nitidus Eggens, 1940, Chramesus

Arb. morph. tax. Ent. 7, 125

Holotypus, Paratypen, Insel Martinique (St. Pierre), 1901, leg. Fleutiaux. Holotypus in Coll. Fleutiaux, Paratypen in Coll. Fleutiaux, Eggers und im SDEI. SDEI: 1 Paratypus, Martinique, St. Pierre, 1901, FleUTIAUX.

opacithorax SchedL, 1940, Phloeborus

Arb. morph. tax. Ent. 7, 205-206

Mehrere Syntypen, Guatemala, Panzos, durch STAudinGER \& BANG-HAAs, im SDEI und in Coll. SchedL.

SDEI: 1 Syntypus, Guatemala, Panzos, durch STAUDINGER \& BANG-HAAS.

opacus Broun, 1895, Acrantus

Ann. Mag. Nat. Hist. (6) 15: 405-419

Mehrere Syntypen, New Zealand, Mount Te Aroha, discovered by Mr. J. H. LEwis.

SDEI: 1 Syntypus bezettelt mit „Acrantus opacus, N. Seeland", handschr. Strohmeyer.

orientalis Eggers, 1943, Camptocerus

Arb. morph. tax. Ent. 10, 244-245

Holotypus, mehrere Paratypen, Brasil, Salobro, Prov. de Bahia, 6.VII.1885, leg. Gounelle, Coll. Strohmeyer, im SDEI und in Coll. EgGERs.

SDEI: Holotypus, 3 Paratypen, mit den Angaben der Beschreibung.

orientalis EGGERS, 1910, Eccoptogaster

Dtsch. ent. Ztschr. 1910, 557-558

Mehrere Syntypen, Kaukasus, Elisabetpol, leg. BABADJANIDES.

SDEI: $1 \sigma^{\star}, 5$ q Syntypen, mit den Angaben der Beschreibung, Coll. v. Heyden.

papuanus Eggers, 1923, Ozopemon

Zool. Meded. Mus. Leiden 7, 157-158

Holotypus, 1 Ex. Südost-Neuguinea, Paumomufluß, leg. Loria, im Museum Genova und in Coll. Eggers; mehrere Ex. Deutsch-Neuguinea, vom Kaiserin Augustafluß, 
im Museum Berlin; 2 Ex. Key-Inseln, leg. Siebers, im SDEI: Paratypen.

SDEI: 1 Paratypus, Key-Eil., Toeal, III.-V.1922, leg. SIEBERS.

\section{paraguayensis EgGeRs, 1930, Dendrosinus}

Ent. Bl. 26, 168

Holotypus, Paraguay, leg. Fiebrig, in Coll. Eggers; 2 Paratypen, Brasil, São Paulo, P. Epitacio oder Tiberisa am Parana, 21.-23.X.1926, leg. OHAus, im Museum Hamburg und in Coll. EgGERs.

SDEI: 1 Holotypus, Paraguay, 6.VIII.1914, leg. FiEBRIG.

\section{pardous EgGers, 1943, Xyleborus}

Arb. morph. tax. Ent. 10, 247-248

+ Holotypus, Brasil, São Paulo, Tal des Rio Pardo, XII.1898, leg. Gounelle, Coll. Strohmeyer, im SDEI.

\section{petzi ReITTER, 1901, Thamnurgus}

Wien. ent. Ztg. 20, 182

Mehrere Syntypen, Ober-Oesterreich bei Gross-Dirn, leg. Petz.

SDEI: 4 Syntypen, Austr. sup., Gr. Dirn, Mont, leg. Petz, Coll. Leonhard, Coll. Kraatz, Coll. v. Heyden.

\section{philippinensis StrohmeYeR, 1911, Sphaerotrypes}

Philipp. Journ. 6, 18-20, Fig. 1, 2, Taf. 1

Holotypus, 1 Paratypus, Mindanao, Zamboanga, P. I., leg. Hutchinson, No. 8849, in der Entomologischen Sammlung des Bureau of Science, Manila, P. I. und in Coll. STROHMEYER.

SDEI: 1 Paratypus, Philippinen, Mindanao, Zamboanga, leg. Hutchinson, No. 8849, Lot Bu. of Sci. P. I., Coll. Strohmeyer. (3 weitere Ex. vom selben Fundort, auch als Paratypen ausgezeichnet.)

\section{plaumanni SCHEDL, 1963, Cnesinus}

Reichenbachia 1, 220

Holotypus, mehrere Paratypen, Brasilien, Santa Catarina, Nova Teutonia, XII.1955/IV.1956/ X.1961, leg. PlaUMann, in Coll. Schedl, Coll. Plaumann und im SDEI. SDEI: $20^{*}, 2$ ㅇ Paratypen, Brasil., S. Catarina, Nova Teutonia, XII.1955/IV.1956, leg. Plaumann.

\section{plaumanni SCHEDL, 1937, Pterocyclon}

Arb. morph. tax. Ent. 4, 68-69

Mehrere Syntypen, Brasilien, Nova Teutonia, leg. PlaUMAnN, im SDEI, in Coll. Schedl und Coll. Plaumann. SDEI: 2 Syntypen, Brasilien, Nova Teutonia, 20.V.1935, leg. Plaumann.

\section{plaumanni Wo0d, 2007, Scolytus}

Bark and ambrosia beetles of South America (Coleoptera, Scolytidae). Brigham Young University, M.L. Bean Life Science Museum; Provo, Utah, 900 p., Beschr. p. 229.

Holotypus, 1 Paratypus, Brasilien, Nova Teutonia, III.1941, leg. F. Plauman im SDEI. posticestriatus EgGeRs, 1939, Xyleborus

Arb. morph. tax. Ent. 6, 119-120

Holotypus, mehrere Paratypen, Formosa, Taihoku, 10.VII.1934/20.VII.1936, leg. Chujô, in Coll. Eggers, Coll. CHujô und im SDEI.

SDEI: 1 , Paratypus, Formosa, Taihoku, 20.VII.1936, leg. CHujô.

\section{primus Wichmann, 1915, Polygraphus}

Ent. Bl. 11, 217

2 Syntypen, Kamerun, im SDEI.

psaltes Hagedorn, 1909, Bothryperus

Dtsch. ent. Ztschr. 1909, 742, Fig. 37

2 Syntypen, Kamerun, leg. ConRADT, im SDEI.

SDEI: 1. Syntypus verloren gegangen, 2. Syntypus nicht im SDEI, nach Gebien im Mus. Hamburg.

\section{pseudoacuminatus ScHedL, 1935, Scolytodes}

Arb. morph. tax. Ent. 2, 51

Mehrere Syntypen, Brasilien, Saude bei São Paulo, 27.IX.1921, leg. Melzer, im SDEI und in Coll. SchedL. SDEI: 22 Syntypen, mit den Angaben der Beschreibung.

\section{pseudobicolor Eggens, 1940, Hexacolus}

Arb. morph. tax. Ent. 7, 132-133

Holotypus, mehrere Paratypen, Guadeloupe, Trois-Riviéres, leg. Dufau, in Coll. Eggers, im Museum Paris und SDEI.

SDEI: 1 Paratypus, mit den Angaben der Beschreibung, 1904, ex Museum Paris.

\section{pseudoimpax SCHEDL, 1954, Hylocurus}

Dusenia 5, 30-31

Mehrere Syntypen, Brasilien, Nova Teutonia, X.1939, leg. Plaumann, in Coll. Schedl und Coll. Plaumann. SDEI: 2 Syntypen, mit den Angaben der Beschreibung.

\section{quadrisulcatus StroHmeYeR, 1908, Dryocoetes}

Ent. Wochenbl. Leipzig 25, Nr. 16, 72-73, Fig. 6, 7

Holotypus, Kashmir, 1905, leg. Rost, in Coll. StrohMEYER, im SDEI.

\section{retrusus SCHEDL, 1940, Xyleborus}

Arb. morph. tax. Ent. 7, 208

Mehrere Syntypen, Kamerun, aus Baumwolle oder Kakao, im SDEI und in Coll. SchedL.

SDEI: 43 Syntypen, mit den Angaben der Beschreibung.

\section{rufopiceus Broun, 1881, Inosomus}

New Zealand Journ. Sci. 2, 739-740

Mehrere Syntypen, New Zealand, nahe Wellington, leg. STEWART.

SDEI: 1 Syntypus, N. Seeland. 
rugicollis SCHEDL, 1940, Hexacolus

Arb. morph. tax. Ent. 7, 205

Mehrere Syntypen, Costa Rica, Hamburgfarm, Ebene Limon, 25.IX.1936, Coll. Nevermann, im SDEI und in Coll. SCHEDL.

SDEI: 1 Syntypus, mit den Angaben der Beschreibung, unter loser Rinde.

samoanus EgGERS, 1928, Dryocoetes

Ent. Bl. 24, 174

2 Syntypen, Apia, leg. Friedrichs, im SDEI und in Coll. EGGERS.

SDEI: 1 Syntypus, Samoa, Apia.

sardus Strohmeyer, 1912, Dryocoetes

Ent. Bl. 8, 57

ca. 200 Syntypen, Sardinien, Sorgono.

SDEI: 9 Syntypen, Sardinien, Coll. Strohmeyer, Coll. v. HEYdEN.

sardus Eggers, 1912, Thamnurgus

Ent. Bl. 8, 114-115

Mehrere Syntypen; Sardinien, Sasali, in Coll. Dodero und Eggers; Asuni, leg. Krausse, in Coll. Loeonhard, EGGERS und WICHMANN.

SDEI: 1 o $^{*}, 1$ \& Syntypen, Sardinia, Assuni, leg. Krausse, Coll. LEONHARD.

schenklingi HagedoRn, 1909, Chlortastus

Dtsch. ent. Ztschr. 1909, 737-738, Fig. 32

Mehrere Syntypen, Kamerun, leg. ConRadT, im SDEI.

SDEI: 11 Syntypen, Kamerun, leg. ConradT, Coll. KRAATZ.

\section{schreineri EICHHOFF, 1881, Cryphalus}

Die europ. Borkenk., 185-187

Mehrere Syntypen, Hinterpommern, leg. Schreiner.

SDEI: 9 Syntypen, Pommern, leg. SchreIner, Coll. KrAATZ.

scobinatus HAGEDORN, 1910, Xyleborus

Dtsch. ent. Ztschr. 1910, 8

Holotypus, Kamerun, leg. Conradt, im SDEI [Coll. KRAATZ].

sereinuus EGgers, 1923, Xyleborus

Zool. Meded. Mus. Leiden 7, 187

Mehrere Syntypen, Insel Mentawei, Sereinu und Si Oban, leg. Modigliani, im SDEI, Museo Civico Genova und in Coll. EgGers.

SDEI: 1 Syntypus, Mentawei, Sipora, Sereinu, V.-VI.1894, leg. Modigliani, Coll. KraAtz.

serrifer HAGEDORN, 1909, Chortastus

Dtsch. ent. Ztschr. 1909, 739

Holotypus, Kamerun, leg. Conradt, im SDEI [Coll. KrAATZ]. setosus EichHOFF, 1867, Hypoborus

Berl. ent. Ztschr. 1867, 391

20 Syntypen, Schleswig-Holstein, leg. EichHOFF.

SDEI: 1 Syntypus, Schleswig-Holstein, Coll. KraAtz.

siculus Eggers, 1908, Taphrorychus

Naturalista Siciliano, 1 (Separatabdruck)

Mehrere Syntypen, Sicilia, Madonie, leg. Ragusa, in Coll.

RAGUSA und Coll. Eggers.

SDEI: 2 Syntypen, Sicilia, Coll. STIERLIN.

siculus EGGeRs, 1912, Thamnurgus

Ent. Bl. 8, 115

Mehrere Syntypen, Sizilien, bei Ficuzza, Sommer 1906, leg. Krüger, in Coll. Leonhard und Coll. Eggers.

SDEI: $10^{\star}, 2$ i Syntypen, mit den Angaben der Beschreibung.

signatus Strohmeyer, 1909, Phloeoborus

Ent. Bl. 5, 248-249

i Holotypus, Brasilia, Jatahy, in Coll. Strohmeyer [Goyaz].

similis Hagedorn, 1908, Dactylipalpus

Dtsch. ent. Ztschr. 1908, 371-372, Fig. 3, 4

Holotypus, Kamerun, Buea, leg. Horn, im SDEI.

similis EgGERS, 1911, Dryocoetes

Ent. Bl. 7, 121-122

Holotypus, Corsica, Vizzavona, leg. Leonhard, in Coll. LEONHARD, im SDEI.

sinensis SCHEDL, 1953, Phloeosinus

Ent. Bl. 49, 23

Mehrere Syntypen, China, Fukien, Kuatun, 28.IV., 4.V. 1946, leg. Tschung Sen, in Coll. Klapperich und Coll. SCHEDL.

SDEI: $10^{\star}$ Syntypus, mit den Angaben der Beschreibung, 28.IV.1946.

siobanus Eggers, 1923, Xyleborus

Zool. Meded. Mus. Leiden 7, 186

Mehrere Syntypen, Insel Mentawei, Si Oban und Sipora, leg. Modigliani, im Museo Civico Genova, in Coll. EGGERS und im SDEI.

SDEI: 1 Syntypus, Mentawei, Si Oban, IV.-VIII.1894, leg. Modigliani, Coll. KraAtz.

siporanus Hagedorn, 1910, Xyleborus

Dtsch. ent. Ztschr. 1910, 11

Mehrere Syntypen, Sipora in Mentawei, im SDEI SDEI: 2 Syntypen, Mentawei, Sipora, Sereinu, V.-VI.1894, leg. Modigliani, Coll. KraAtz. 
sirambeanus HAGEDORN, 1910, Ozopemon

Dtsch. ent. Ztschr. 1910, 3; als var. von theklae HAGEdorN beschrieben

Zahlreiche Syntypen, Sumatra, Si-Rambé, leg. ModIGLIANI, im SDEI.

SDEI: 2 Syntypen, Sumatra, (Mentawei), Si Rambe, 12.1890-3.1891, leg. ModigLIANI

\section{sisyrnophorus HAGEDORN, 1910, Xyleborus}

Dtsch. ent. Ztschr. 1910, 7-8

Holotypus, Tandjong Morawa, im SDEI [Coll. ScHUlTHEISS].

\section{spinosus Hagedorn, 1909, Adiaeretus}

Dtsch. ent. Ztschr. 1909, 745, Fig. 41

1 Ex. Caffraria, in Coll. Hagedorn; 1 Ex. Transvaal, leg. HARTMANN, im SDEI: Syntypen.

SDEI: 1 Syntypus, Transvaal, leg. Hartmann, Coll. KRAATZ.

\section{squamulosus Strohmeyer, 1911, Cryphalus}

Philipp. Journ. 6, 20-21, Fig. 3

Holotypus, No. 1420, 1 Paratypus, Mindoro, Calapan, P. I., leg. WeBb, in der Entomologischen Sammlung des Bureau of Science Manila, P. I. und in Coll. StrohMEYER.

SDEI: 1 Paratypus, mit den Angaben der Beschreibung, Coll. Strohmeyer, Acc. No. 1420, Lot, Govt. Lab. Coll.

stebbingi StrohmeYer, 1908, Ips

Ent. Wochenbl. Leipzig 25, Nr. 16, 69, Fig. 2, 3

4 o Syntypen, Himalaya occidentalis, Kulu, Cedrus deodara, leg. Rost, in Coll. Strohmeyer.

SDEI: Syntypen, Himalaya, Kulu, 1906, leg. Rost, Cedrus deodara, Coll. Strohmeyer, Coll. v. Heyden. (6 weitere Ex. vom selben Fundort, auch als Syntypen ausgezeichnet).

\section{stierlini Eggers, 1911, Cryphalus}

Ent. B1. 7, 121

2 Syntypen, Italia, in Coll. StierLin und Coll. Eggers. SDEI: 1 Syntypus, Italia, Coll. EgGERs.

\section{stoeckleini SCHEDL, 1935, Phloesinus}

Arb. morph. tax. Ent. 2, 241

Mehrere Syntypen, Dalmatien, Makarska, leg. SтöckLEIN, im SDEI und in Coll. SCHEDL.

SDEI: $1 \sigma^{\star}, 1$ \& Syntypen, Dalmatien, Makarska.

\section{striatum Eggers, 1940, Hylocurosoma}

Arb. morph. tax. Ent. 7, 139-140, Fig. 1

Holotypus, mehrere Paratypen, Guadeloupe, Trois-Riviéres, 1904, leg. Dufau, im Museum Paris, SDEI und in Coll. EgGers.

SDEI: 1 Paratypus, mit den Angaben der Beschreibung, ex Museum Paris. submarginatus SCHEDL, 1937, Scolytus

Arch. Inst. Biol. veg. Rio de Jan. 3, 163

o ㅇ Syntypen, Brasilien, Nova Teutonia, 12.1934, leg. Plaumann und Paraguay, Hohenau, leg. Jаков; im SDEI und Coll. SCHEDL.

SDEI: 1 Syntypus, Paraguay, Hohenau, leg. ЈАсов.

\section{subsimilis ScHedL, 1956, Pityophthorus}

Ztschr. angew. Ent. 38, 25

o \& Syntypen, Guatemala, G. Ciudad, 1500 m, 14.+ 25.8.1951, aus Pinus tenuifolia, leg. Schwerdtfeger, in Coll. SCHEdL und SCHWERDTFEGER.

SDEI: 3 Paralectotypen, Guatemala, Ciudad, Pin. tenuifolia, 25.8.1951, leg. Schwerdtfeger (Lectotypenfestlegung durch BRIGHT; Coleopterists Bull. 30, 187; 1976).

\section{substriatus StROHMEYER, 1914, Hylastes}

Ent. Bl. 10, 7-8, Fig.

2 Syntypen, Turkestan, Ala-Tau-Gebirge, Wernyi, Kuldscha, Juldus, in Coll. Strohmeyer, im SDEI.

\section{subsulcatus SCHEDL, 1963, Breviophthorus}

Reichenbachia 1, 223

Holotypus, 2 Paratypen, Brasilien, Paraná, Rondon, 24., 27., 30.X.1952, leg. Plaumann, in Coll. Schedl, Coll. PLAUMANN und im SDEI.

SDEI: 1 Paratypus, mit den Angaben der Beschreibung, 24.X.1952.

\section{sulcatus ScHedL, 1959, Breviophthorus}

Beitr. Ent. 9, 552-553

Holotypus, mehrere Paratypen, Brasilien, Matto Grosso, Rio Caraguata, V., VI., 5.IX.1953, leg. PlaumanN, in Coll. Schedl, Coll. Plaumann und im SDEI.

SDEI: 2 Paratypen, Brasilien, Rio Caraguata, $21^{\circ} 48^{\circ}$ B., $52^{\circ} 27^{\circ}$ L., $400 \mathrm{~m}$, IV., 19.IV.1953, leg. Plaumann, $400 \mathrm{~m}$.

\section{sumatranus StrohmeYer, 1908, Acanthotomicus}

Ent. Wochenbl. Leipzig 25, Nr. 16, 69-70, Fig. 4, 5

$0^{\star}$ Holotypus, Sumatra, Palembang, in Coll. StroHMEYER, im SDEI.

\section{sumatranus EgGERS, 1927, Hylurgulus}

Treubia 9, 393: nom. praeocc. Phloeosinus hylurgulus EgGERs. 2 Ex. Süd-Sumatra, Wai Lima, im Museum Buitzenzorg und in Coll. Eggers; 3 Ex. Tenasserim, Coll. Helfer, 1830, im Museum Prag und in Coll. EgGERs: Syntypen. SDEI: 1 Syntypus, S. Sumatra, Wai Lima, Lampongs, No. 112, XI-XII.1921, leg. KARNY.

\section{tachygraphus SAHLBERG, 1834, Bostrichus}

Insecta fennica 1 (2), 152-153

Mehrere Syntypen, Fennia australi.

SDEI: 2 Syntypen, Fenn. austr., Coll. KraATZ. 
tanganus SCHEDL, 1937, Coccotrypes

Arb. morph. tax. Ent. 4, 67-68

Mehrere Syntypen, Aegypten, Alexandria, in SteinnußKnöpfen, ded. Herfs, im SDEI und in Coll. SchedL. SDEI: 6 Syntypen, Aegypten.

\section{tectus Eggers, 1943, Camptocerus}

Arb. morph. tax. Ent. 10, 245-246

Holotypus, mehrere Paratypen, Brasil, Serra da Bernada, Pernambuco, 4.VI.1894, leg. Dunant, Coll. Strohmeyer, im SDEI und in Coll. EGgERs.

SDEI: Holotypus, 2 Paratypen, mit den Angaben der Beschreibung, 5.VI.1894.

testudo Eggers, 1939, Xyleborus

Arb. morph. tax. Ent. 6, 116-118

Holotypus, mehrere Paratypen, Formosa, Trichu, XI.1930, leg. Mitino, in Coll. Chujô, Coll. Eggers und im SDEI.

SDEI: 1 ㅇ Paratypus, mit den Angaben der Beschreibung, 9.XI.1930.

theklae Hagedorn, 1910, Ozopemon

Dtsch. ent. Ztschr. 1910, 2, Fig. 43

Mehrere Syntypen, Sumatra, leg. Modigliani, im SDEI.

SDEI: 5 Syntypen, Sumatra, Si-Rambé, XII.1890-III.1891, leg. Modigliani, Coll. KraAtz.

thomsoni Ferrari, 1867, Cryphalus

Die forst- und baumzuchtschädl. Borkenk., 12, 14-16

7 Syntypen, Europa, Umgebung Jülich.

SDEI: 1 Syntypus, Jülich.

trispinosus Strohmeyer, 1908, Scolytus

Ent. Wochenbl. Leipzig 25, Nr. 16, 69, Fig. 1

o Holotypus, Japan, Kushiro, leg. Rost, in Coll. StrohMEYER, im SDEI.

tropicus HAGEDORN, 1910, Xyleborus

Dtsch. ent. Ztschr. 1910, 12

Mehrere Syntypen, Kamerun, leg. ConRadT, im SDEI.

SDEI: 1 Syntypus, Kamerun, leg. Conradt, Coll. KRAATZ.

tuberculatus HageDORN, 1909, Diamerus

Dtsch. ent. Ztschr. 1909, 734, Fig. 38

Holotypus, Kamerun, leg. Conradt, im SDEI [Coll. KRAATZ].

tuberculatus Strohmeyer, 1912, Ozopemon

Ent. Mitt. 1, 38-39

2 Syntypen, Formosa, leg. Sauter, in Coll. Strohmeyer, im SDEI. unimodus SCHEDL, 1959, Cryptocleptes

Beitr. Ent. 9, 549-550

Holotypus, mehrere Paratypen, Brasilien, Matto Grosso, Rio Caraguata, III.1953, leg. Plaumann, in Coll. SchedL, Coll. Plaumann und im SDEI.

SDEI: $10^{\star}, 1$ ㅇ Brasilien, Rio Caraguata, Matto Grosso, III.1953, leg. PlaumanN; 1 Ex. dito, 21.III.1953; 1 + , dito, IV.1953: Paratypen.

ursinus HAgEDORN, 1908, Xyleborus

Dtsch. ent. Ztschr. 1908, 381, Fig. 26, 27

Holotypus, Sumatra, Si-Rambé, leg. Modigliani, im SDEI [XII.1890-III.1891, Coll. KraATZ].

ventralis Sharp, 1877, Pachycotes

Ent. Monthly. Mag. 14, 10

Mehrere Syntypen, New Zealand, Riccarton, leg. WAKEFIELD.

SDEI: 1 Syntypus, New Zealand.

vismiae Eggers, 1929, Loganius

Wien. ent. Ztg. 46, 63-64

Holotypus, mehrere Paratypen, Costa Rica, La Caja, 8 km westlich San José, leg. H. SCHMIdT, im SDEI und in Coll. EGGERs.

SDEI: $\sigma^{\top}$ Holotypus, 16 Paratypen, mit den Angaben der Beschreibung.

willei SCHEDL, 1937, Phloeotribus

Arb. morph. tax. Ent. 4, 66-67

Mehrere Syntypen, Peru, Lima, 15.IX.1935, Coll. WiLle, im SDEI und in Coll. ScHEDL.

SDEI: 1 Syntypus, mit den Angaben der Beschreibung, Nr. 305-135.

Wahrscheinlich nicht beschriebene Arten

acaciae SCHEDL, Phloeosinus

SDEI: 2 Typen, Congo Belge, Hembe-Bitale, 18.VIII.1952, leg. SCHEDL.

aethiopicus Strohmeyer, Phloeoborus

SDEI: 6 Typen, Abessinien, leg. Kristensen, Coll. STROHMEYER.

barbatus StROHMEYER, Gnathotrichus

SDEI: 3 Typen, Chile, Coll. StrohmeYer.

curvidens StROHMEYER, Gnathotrichus

SDEI: 2 Typen, Chile, Coll. Strohmeyer. 
niger StROHMEYER, Hexacolus

SDEI: 1 Type, Guadeloupe, Coll. Strohmeyer.

nudigranis APfelBeCK, Pityophthorus

SDEI: 2 Typen, Trebevi..., V. 1920.

opacum Strohmeyer, Ctonoxylon

SDEI: 1 Type, Kamerun.

submarginatus SCHEDL, Scolytus

SDEI: 1 Type, Paraguay, Hohenau, leg. ЈАсов.

subsimilis SCHEDL, Pityophthorus

SDEI: 3 Typen, Guatem., Cindat. Pin. tenuif., 5.VIII.1951, leg. SCHWERDTFEGER. sumatranus EgGERS, Erineosinus

SDEI: 3 Typen, Sumatra, 7.VIII.1924, an Blütenstiel einer Euphorbia cea.

tuberculatus EICHHORST, Hylastes

SDEI: 8 Typen, Ostsibirien, Chabarofka.

tuberculosus Strohmeyer, Chortastus

SDEI: 1 Type, Kamerun, Coll. Strohmeyer. 